\title{
Automating the E-learning Personalization
}

\author{
Fathi Essalmi ${ }^{1}$, Leila Jemni Ben Ayed ${ }^{1}$, Mohamed Jemni ${ }^{1}$, \\ Kinshuk $^{2}$, and Sabine Graf ${ }^{2}$ \\ ${ }^{1}$ The Research Laboratory of Technologies of Information and Communication \& Electrical \\ Engineering (LaTICE), Higher School of Sciences and Technologies of Tunis (ESSTT), \\ University of Tunis, Tunisia \\ ${ }^{2}$ School of Computing and Information Systems, Athabasca University, Canada \\ fathi.essalmiaisg.rnu.tn, leila.jemni@fsegt.rnu.tn, \\ mohamed.jemni@fst.rnu.tn, kinshuk@athabascau.ca, \\ sabineg@athabascau.ca
}

\begin{abstract}
Personalization of E-learning is considered as a solution for exploiting the richness of individual differences and the different capabilities for knowledge communication. In particular, to apply a predefined personalization strategy for personalizing a course, some learners' characteristics have to be considered. Furthermore, different ways for the course representation have to be considered too. This paper studies solutions to the question: How to automate the E-learning personalization according to an appropriate strategy? This study finds an answer to this original question by integrating the automatic evaluation, selection and application of personalization strategy. In addition, this automation is supported by learning object metadata and an ontology which links these metadata with possible learners characteristics.
\end{abstract}

Keywords: Personalization strategy, E-learning, Evaluation of personalization parameters.

\section{Introduction}

This research originated from the recognition of the need of a complete solution to the central question: How to automate the E-learning personalization according to an appropriate strategy? Through a comprehensive literature review, [1] identified 16 personalization parameters ${ }^{1}$ and 23 personalization systems implementing 11 personalization strategies ${ }^{2}$. The different reported strategies express different personalization needs. For example, PERSO uses Case Based Reasoning (CBR) approach to determine which course to propose to the students based on their levels of

\footnotetext{
${ }^{1}$ A personalization parameter includes a set of complementary learners' characteristics. For example, the learner's level of knowledge (includes the learners' characteristics beginner, intermediate and advanced), motivation level (includes the learners' characteristics low and high motivation) and the active/reflective dimension of the Felder-Silverman learning style model are personalization parameters.

${ }^{2}$ A personalization strategy includes a set of personalization parameters.
} 
knowledge and their media preferences [2]. MetaLinks, an authoring tool and web server for adaptive hyperbooks, has been used to build a geology hyperbook [3]. MetaLinks uses three personalization parameters, namely learner's level of knowledge, learning goals and media preferences. AHA! [4] uses stable presentations, adaptive link (icon) annotations and adaptive link destinations for personalizing Elearning. The personalization strategy of AHA includes the parameters FelderSilverman learning style, media preference and navigation preference. Milosevic, Brkovic, and Bjekic [5] used Kolb's learning cycle for tailoring lessons. Their work also incorporated the learner motivation as a personalization parameter, which is used to determine the complexity and the semantic quantity of learning objects. Others personalization systems implementing personalization strategies are reported in the literature. PASER [6] has been developed for course planning according to learners' goals and their level of knowledge, using a domain ontology which describes a hierarchy of the artificial intelligence area. Protus [7] considers the Felder-Silverman Learning Styles Model and the learner's level of knowledge to recommend relevant links and activities for learners. [8] uses Web mining techniques to deliver appropriate content to learners according to their interests and needs. The number of theoretical and possible personalization strategies, that can be used for personalization, is very high (>50000) [1]. This high number expresses a richness of the E-learning personalization domain that could be exploited by automating the E-learning personalization according to appropriate strategy. Personalizing all courses according to only one predefined personalization strategy would not fit the specificities of courses [9] and teachers' preferences [1]. Therefore, we need to select and apply the appropriate personalization strategy for each course.

This paper answers to the research question: How to automate the E-learning personalization according to an appropriate strategy? This central question could be divided into three sub-questions. (1) How to automate the selection of the appropriate personalization strategy? (2) How to automate the design of personalized learning scenarios? and, (3) How to integrate the solutions to the above mentioned two subquestions?

Some parts of the central question have already been solved. In particular, the first sub-question (how to automate the selection of an appropriate personalization strategy?) has been studied in [2], where an approach has been presented for the automatic evaluation of personalization strategies. Metrics evaluating personalization strategies are supported by an Ontology representing and managing the Semantic Relations between Values of Data elements and Learners' characteristics (OSRVDL). The second sub-question (how to automate the design of personalized learning scenarios?) has also been partially studied. In particular, a manual (not automated) solution to this second sub-question has been presented in [1] where design and experiment of an architecture for the personalization have been proposed in two complementary levels: the E-Learning Personalization level 1 (ELP1), and the ELearning Personalization level 2 (ELP2). ELP1 allows for the personalization of learning contents and structure of the course according to a given (specified within ELP2) personalization strategy. ELP2 allows for defining the personalization strategy flexibly. This level of personalization enables teachers to select the learning scenario and to specify manually the personalization strategy (to be applied on the selected learning scenario) by choosing a subset of personalization parameters. To achieve the 
integrated solution of the central question, the following gaps need to be studied. The answer to the second sub-question (how to automate the design of personalized learning scenarios) is not yet fully resolved (automated). This paper integrates the ontology OSRVDL with ELP1+ELP2 to automatically apply the selected personalization strategy. This ontology allows for automatically generating the learning objects appropriate to learners' characteristics included in the selected personalization strategy. Another gap still remaining is the third sub-question, namely how to integrate the automatic evaluation, selection and application of personalization strategy. This paper provides answer to this question by integrating metrics evaluating personalization strategies with ELP1+ELP2+OSRVDL.

The next section of the paper presents the approach for automatic design of personalized learning scenarios and evaluation of personalization strategies. Section 3 presents an integrated framework for automating the E-learning personalization according to the appropriate strategy. Finally, section 4 concludes the paper with a summary of the work, its limitations and potential future research directions.

\section{Automating the Design of Personalized Learning Scenarios and Evaluation of Personalization Strategies}

This section presents two processes which are needed to achieve the central question. Then, section 3 presents an integration of these processes in the whole architecture. The first process is concerned with the automatic design of personalized learning scenarios. This process raises its importance from the need to generate appropriate learning scenario by considering the personalization strategy and the learner profile. The second process is needed to evaluate personalization strategies and help teachers in selecting the appropriate one.

The first process (automatic design of personalized learning scenarios) uses the ontology OSRVDL $[9,10]$ which includes 76 semantic relations between metadata elements and learners characteristics. The richness of the ontology and its extensibility is the basis for an extensible and generic process. This process links learning objects with the appropriate learners characteristics based on OSRVDL. Then, appropriate and non-appropriate learning objects can be used for personalizing E-learning courses. For example, in an adaptive navigational support, appropriate learning objects could be marked with green icons and non-appropriate learning objects could be marked with red icons. If no information is available for the adaptation decision for some learning objects, the adaptation is considered as neutral for those learning objects. This process is based on metadata (which is commonly used for the reuse of learning objects), course, and OSRVDL. For example, if we assume that: (1) there is a semantic relation between the data element 4.1 Format [11] associated with the value image and the learner media preference of graphic; and, (2) a course contains a learning object $\mathrm{O} 1$ which is described by the data element 4.1 Format associated with the value image, we can conclude that the learning object $\mathrm{O} 1$ is appropriate for the media preference graphic (see Figure 1). This process can be used for operationalizing the personalization of courses. In addition, this approach can also be used for the analyses of the metadata describing the learning objects in order to evaluate personalization strategies. 


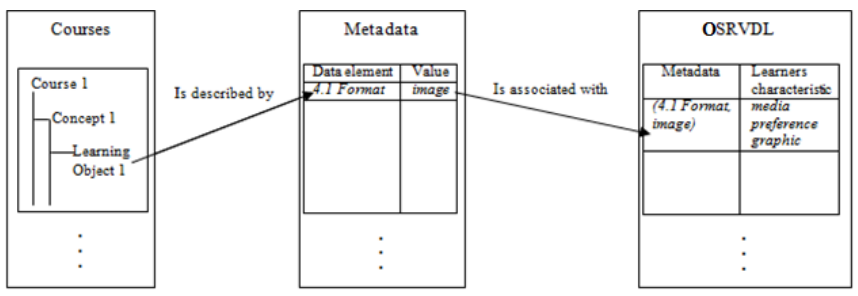

Fig. 1. Appropriate learning object

The second process (evaluation of personalization strategies) benefits from the result of the first process. The generic approach for automatic selection of appropriate learning objects can be used for earlier evaluation of personalization parameters (before starting the learning process and determining the learners' characteristics). This is because of two reasons. The first one is: it is possible to study automatically the feasibility and the easiness of personalizing a given course according to a personalization parameter. For example, when a given course contains learning objects appropriate for each learner's characteristic included in a personalization parameter, the parameter is considered as useful for personalizing the given course. However, if the given course does not contain learning objects appropriate for the learners' characteristics included in another personalization parameter, this parameter is considered as non-useful for personalizing the course. The second reason is the feasibility of comparing personalization parameters. Figure 2 presents the structure of a course and a matrix of appropriate learning objects used for the evaluation of personalization parameters. This matrix contains the personalization parameters and their divergent characteristics in the columns. The rows of the matrix contain the courses and the concepts included in them. Each cell contains the learning objects presenting a specific concept according to a specific characteristic. The last lines of the matrix can include metrics evaluating personalization parameters. For example, one of these metrics calculates, for each personalization parameter, the number of cells which include a learning objects divided by the number of cells. This rate increases when there are more learners' characteristics considered by learning objects. This metric allows for comparing personalization parameters.

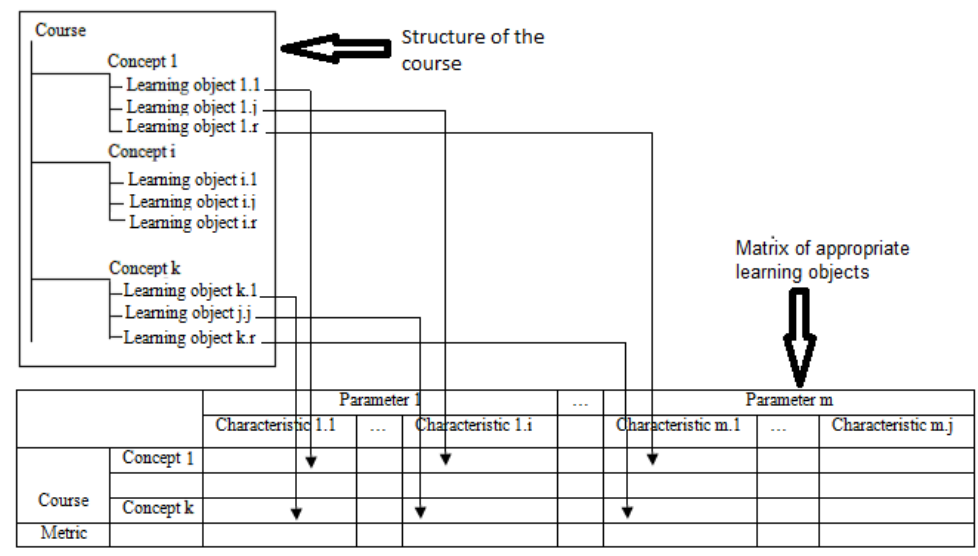

Fig. 2. Early evaluation of personalization parameters 


\section{An Integrated Framework}

This section presents a solution to the third sub-question (how to integrate the automatic evaluation, selection and design of personalized learning scenarios). At first, the basic solution is integrated with that of sub-questions 1and 2. ELP1+ELP2 [1], which consists of a system architecture for the personalization at two complementary levels, is integrated with the ontology OSRVDL, the service evaluating personalization strategy and the service for automating the design of personalized learning scenarios.

ELP1+ELP2 is built by integrating components which focus on the personalization level 1 (ELP1) and the personalization level 2 (ELP2). Furthermore, ELP1 must apply the personalization strategy specified by the teacher in ELP2. ELP1+ELP2 is a new vision of personalization that offers a solution towards some fundamental limitations of E-learning personalization systems. The main advantages of ELP1+ELP2 include the ability of teachers to select the most suitable personalization parameters for their learning scenarios and the possibility of applying more than one personalization parameter according to the specifics of the learning scenarios. The personalization systems available in the literature offer important functionalities for determining the learner characteristics according to a predefined subset of the personalization parameters. The federation of these functionalities and their combination allows for generation of other personalization strategies. However, the personalization systems are developed with different programming languages and tested/used in different contexts. This makes the combination of the functions offered by these systems rather difficult. In this context, the Web services technology offers a powerful solution for the interoperability between multiple applications. In fact, a service can be considered as a distant function which is executed when it is called. In this way, when using services, developers are not interested in the implementation (algorithm, structure, programming language) and the platform of the service. Developers want to only call the service when they need it. Therefore, Web service is an emergent solution for integration of applications. Besides, the personalization systems are tested on different Web servers. This also advocates use of Web services technology for the integration of these personalization systems. Web services technology also offers a major solution for federation of the functionalities of personalization systems. In this context, an important step for concretizing the proposed approach consists of utilizing Web services technology when developing ELP1+ELP2.

The mechanism of ELP2 is based on the Service for Specifying Personalization Strategies (SSPS). SSPS is needed to concretize the new idea of allowing the pedagogues and teachers to specify the personalization strategy adapted for the learning scenario. This service enables the selection of personalization parameters (SPP). For the given courses, the selected personalization parameters and their list of values are stored in a relational database.

ELP1 includes 4 services. The first one is the Service for Specifying and Reusing Learning Scenarios (SSRLS). This service allows the designer of learning scenarios to define a structure of a learning scenario and to determine the content to be communicated to the learners for each component of the defined structure. A learning scenario can be represented in the form of a tree of chapters, subchapters, pedagogical activities, and so on. The second service is the Services for Determining Learners' 
Characteristics (SDLC). The aim of SDLC is to federate the set of services for determining the learners' characteristics where each of them is associated with a personalization parameter. The third service is the Service for Applying Personalization Strategies (SAPS). SAPS allows for the application of the personalization strategy specified in SSPS by combining the learner profile with the learning scenarios. Besides, SAPS is responsible for building the learner profile by gathering the output of the selected services for approximating the required learner characteristics. The fourth service is the Service for Learner Navigational Support (SLNS). SLNS allows for the illustration of the learning content in the form of adaptive navigational support. SLNS displays the structure of learning scenarios designed with SSRLS in an adaptive way.

The integrated framework is presented in the figure 3. ELP1+ELP2 is enhanced by integrating the ontology OSRVDL, the service evaluating personalization strategy and the service for automating the design of personalized learning scenarios.

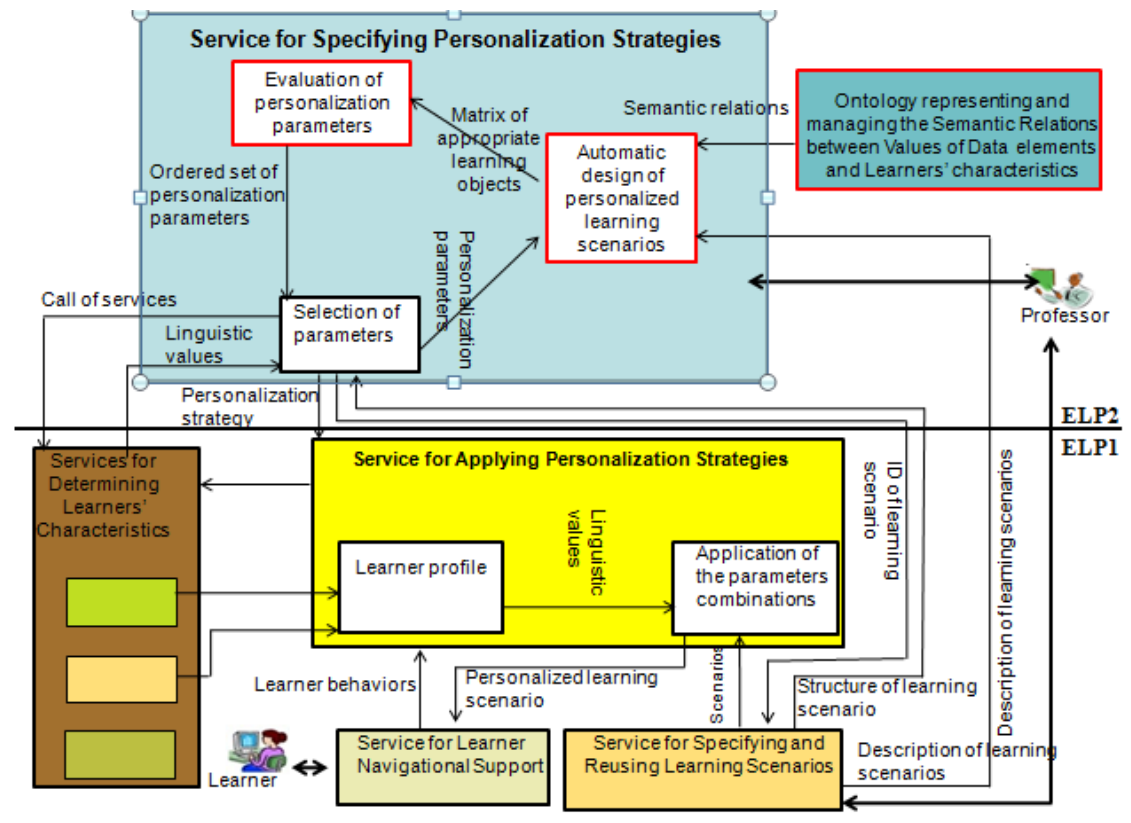

Fig. 3. An integrated framework

The automatic design of personalized learning scenarios plays two roles. The first one is to prepare the matrix of appropriate learning objects as presented in the figure 2. This matrix is used by the component Evaluation of personalization parameters. After the selection of the appropriate personalization parameters based on their evaluation, the automatic design of personalized learning scenarios allows for having learning scenarios appropriate for the selected personalization strategy and the learner profile. This is done by considering only those columns of the matrix which include the selected personalization parameters. 


\section{Conclusion, Limitation and Potential Future Research Directions}

There is a rich set of personalization strategies which could help for the success of Elearning. These personalization strategies need to be evaluated to select the appropriate one for each course. Furthermore, personalized learning scenarios need to be designed based on the selected personalization strategy. These processes (evaluation of personalization strategy and design of personalized learning scenarios) need to be automated and integrated in order to reduce the efforts and times of course personalization.

This paper presented a solution to the central question: How to automate the Elearning personalization according to an appropriate strategy? An integrated framework is presented for the personalization of E-learning at two levels (ELP1 and ELP2), evaluation of personalization parameters, and automatic design of personalized learning scenarios.

ELP1 is considered as a generalization of the E-learning personalization. ELP1 allows for applying any specific personalization strategy when appropriate learning scenarios are designed. ELP2 supports teachers in selecting the learning scenario and in specifying the personalization strategy (to be applied on the selected learning scenario). This approach enables the application of the declared personalization strategies without developing a personalization system for each possible personalization strategy [1].

The evaluation of personalization parameters can be used to compare and select appropriate personalization parameters for personalizing each course. For the automation of the evaluation process, metrics such as the rate of learning objects appropriate for learners' characteristics are used. These metrics are included in ELP2. The evaluation of personalization parameters was supported by 76 Semantic Relations between Values of Data elements and Learners' characteristics stored in OSRVDL [9, 10].

Concerning the automatic design of personalized learning, the proposed approach exploits learning objects annotated with Learning Object Metadata (LOM) standard and semantic relations between data elements and learners' characteristics in order to determine learning objects appropriate for learners' characteristics.

The proposed approach has a limit which concerns the availability of the Services for Determining Learners' Characteristics (SDLC). For the application of personalization strategies, a Web service is needed for each personalization parameter. For some personalization parameters, Web services are implemented and used for the evaluation of the proposed approach. Other personalization parameters are reported in the literature without publication of Web service (or software components) for determining learners' characteristics. The absence of published Web service for each personalization parameter is a constraint towards an easy specification of personalization strategies. It might be interesting to collaborate with the research structures working on these parameters for the capitalization of the developed components (for determining learners' characteristics) by their implementation and publication as Web services. In this way, each component could be used/called by several personalization systems.

Beside the future works for reducing the limit of the proposed approach, there are other potential future works concerning ELP1+ELP2 and OSRVDL that deserve some consideration. 
Concerning ELP1+ELP2, ELP3 should be studied as an additional layer of the Elearning personalization. ELP3 symbolizes the E-learning systems which support the personalization by educational institutes as personalization logistics according to the personalization needs and environments.

Concerning OSRVDL, future directions of this research should deal with extending OSRVDL for describing the Web services implementing the personalization parameters (including the URL of the Web service, available functions, organizations, researchers working on the personalization parameters, and so on). This extension could facilitate the reuse of the personalization parameters. Furthermore, OSRVDL should be extended by considering additional data elements, learners' characteristics and semantic relations between them.

\section{References}

1. Essalmi, F., Jemni Ben Ayed, L., Jemni, M., Kinshuk, Graf, S.: A fully personalization strategy of E-learning scenarios. Computers in Human Behavior 26(4), 581-591 (2010)

2. Chorfi, H., Jemni, M.: PERSO: A System to customize e-training. In: 5 th International Conference on New Educational Environments, Lucerne, Switzerland, (May 26-28, 2003)

3. Murray, T.: MetaLinks: Authoring and affordances for conceptual and narrative flow in adaptive hyperbooks. International Journal of Artificial Intelligence in Education 13, 199-233 (2003)

4. Stash, N., Cristea, A., de Bra, P.: Adaptation to Learning Styles in ELearning: Approach evaluation. In: Reeves, T., Yamashita, S. (eds.) Proceedings of World Conference on E-Learning in Corporate, Government, Healthcare, and Higher Education, pp. 284-291. AACE, Chesapeake (2006)

5. Milosevic, D., Brkovic, M., Bjekic, D.: Designing lesson content in adaptive learning environments. International Journal of Emerging Technologies in Learning 1(2) (2006)

6. Kontopoulos, E., Vrakas, D., Kokkoras, F., Bassiliades, N., Vlahavas, I.: An ontology based planning system for e-course generation. Expert Systems with Applications 35, 398-406 (2008)

7. Klasnja-Milicevic, A., Vesin, B., Ivanovic, M., Budimac, Z.: E-Learning personalization based on hybrid recommendation strategy and learning style identification. Computers \& Education 56, 885-899 (2011)

8. Khribi, M.K., Jemni, M., Nasraoui, O.: Toward a Hybrid Recommender System for ELearning Personalization Based on Web Usage Mining Techniques and Information Retrieval. In: Proceedings of World Conference on E-Learning in Corporate, Government, Healthcare, and Higher Education (E-learn 2007), vol. 7(1), pp. 6136-6145 (2007)

9. Essalmi, F., Jemni Ben Ayed, L., Jemni, M.: An ontology based approach for selection of appropriate E-learning personalization strategy, DULP Workshop. In: The 10th IEEE Int. Conf. on Advanced Learning Technologies, Sousse, Tunisia, pp. 724-725 (2010)

10. Essalmi, F., Jemni Ben Ayed, L., Jemni, M., Kinshuk, Graf, S.: Selection of appropriate Elearning personalization strategies from ontological perspectives. Special Issue on the Design Centered and Personalized Learning in Liquid and Ubiquitous Learning Places. Interaction Design and Architecture(s) Journal 9-10, 65-84 (2010)

11. IEEE Inc. Draft Standard for Learning Object Metadata (2002) 